Peer $J$

Organic Chemistry
Submitted 20 May 2019 Accepted 16 July 2019 Published 15 October 2019

Corresponding authors Jesús Díaz, jdal@unex.es Carlos Fernández Marcos, cfernan@unex.es

Academic editor Robert Smith

Additional Information and Declarations can be found on page 11

DOI 10.7717/peerj-ochem.1

Copyright

2019 Bornadiego et al.

Distributed under

Creative Commons CC-BY 4.0

OPEN ACCESS

\section{Direct multicomponent synthesis of benzocoumarins}

\author{
Ana Bornadiego, Jesús Díaz and Carlos Fernández Marcos \\ Laboratory of Bioorganic Chemistry \& Membrane Biophysics (L.O.B.O.), School of Veterinary Sciences, \\ University of Extremadura, Cáceres, Spain
}

\section{ABSTRACT}

A straightforward and versatile protocol for the synthesis of dibenzo[b, $d]$ pyran-6-ones from readily available 3-carbonylcoumarins is reported. Our strategy is based on a reaction cascade of successive [4+1] and [4+2] cycloadditions that occur in one single operation. This work illustrates the unprecedented use of a multicomponent reaction of isocyanides for the preparation of this biologically relevant type of compounds. Notably, in this highly convergent and atom-economic process, one new single and two new double carbon-carbon bonds are formed in a simple synthetic operation.

Subjects Natural Products, Organic Chemistry (other), Organic Compounds, Synthetic Organic Chemistry

Keywords Cycloadditions, Multicomponent reactions, Isocyanides, Benzocoumarins, Natural products, Diels-alder reaction, Tandem reactions

\section{INTRODUCTION}

Coumarins or $2 \mathrm{H}$-chromen-2-ones represent an important class of compounds, which are ubiquitous structures as secondary metabolites in plants and many species of fungi and bacteria. The parent member of this class, coumarin itself, was first isolated by Vogel in 1820 from the tonka bean (Dipteryx odorata) (Vogel, 1820). Since then, many different simple and polycyclic coumarins have been discovered and extensively studied due to their potent and singular biological activities (Stefanachi et al., 2018; Medina et al., 2015; Borges et al., 2005; Lavoie et al., 2019) and their photophysic properties (Wagner, 2009; Trenor et al., 2004). Among them, benzo $[c]$ coumarins (1; Fig. 1) have emerged as privileged structures in drug discovery (Garazd \& Garazd, 2016; Mao et al., 2014). Relevant examples include mycotoxin alternariol (2), (Solfrizzo, 2017) antioxidant and anticancer ellagic acid (3), (Ceci et al., 2018) synthetic cannabinoid agonists cannabilactones (4), (Khanolkar et al., 2007) antimalarial dioncolactone (5) (François et al., 2016) and the glucoside derivatives with antitumor properties gilvocarcins (6), (Tomita, Takahashi \& Tamaoki, 1982) chrysomycins (7), (Matson et al., 1989) and ravidomycins (8; Fig. 1) (Yamashita et al., 1998).

Thus, in the last few years, several research groups have directed their efforts to develop efficient syntheses of benzo[c]coumarins. Most approaches rely upon the construction of $\mathrm{B}$ ring of benzo[c] courmarin (1), either by lactonization of a biaryl (9; Fig. 2, path a) (Morack, Metternich \& Gilmour, 2018; Ramirez, Bosque \& Gonzalez-Gomez, 2015; Luo et al., 2013) or by the formation of biaryl bond starting from a bicyclic ester (10; Fig. 2, path b) (Ortiz Villamizar et al., 2017). These methods usually involve the use of UV light, transition metal catalysts, and toxic oxidizing agents, limiting their actual applicability. Other efficient 
<smiles>O=c1oc2ccccc2c2ccccc12</smiles>

1<smiles>Cc1cc(O)cc2oc(=O)c3c(O)cc(O)cc3c12</smiles>

2 (Alternariol)<smiles>O=c1oc2c(O)c(O)cc3c(=O)oc4c(O)c(O)cc1c4c23</smiles>

3 (Ellagic acid)<smiles>[R]c1cc(O)c2c(c1)oc(=O)c1ccc(C)cc12</smiles>

4 (Cannabilactone)<smiles>COc1cc2c(=O)oc3c4c(ccc3c2c2cccc(O)c12)C[C@@H](C)N[C@H]4C</smiles>

5 (Dioncolactone A)<smiles>[R]c1cc(OC)c2c(c1)c(=O)oc1c2cc(OC)c2c(O)ccc([R])c21</smiles>

6 (Gilvocarcin V) 7 (Chrysomycin V) 8 (Ravidomycin)

Figure 1 The structure of benzo $[c]$ coumarin and some natural and synthetic bioactive analogues. Full-size DOI: 10.7717/peerjochem.1/fig-1

alternative approaches reported in literature involve tandem cycloaddition reactions to form C ring of benzo[c]courmarin 1 (Fig. 2, path c) (Pottie et al., 2011; He et al., 2012). In this latter case, the Diels-Alder reaction is especially useful, as it offers the greatest potential for diversity (Pottie et al., 2011). Unfortunately, harsh reaction conditions and the use of starting materials that are difficult to synthesize are generally required (Pottie et al., 2011).

Herein we describe a facile synthesis of derivatives of benzo[c]coumarins (1a-n) by the Diels-Alder reaction of aminofuranocoumarins (11) prepared in situ from 3carbonylcoumarins (12) and isocyanides (13). This approach has the advantages of an easy and concise construction of 2-aminofuranes by the [4+1] cycloaddition reaction of $\alpha, \beta$-unsaturated carbonyl compounds with isocyanides, (Chatani et al., 2003; Ito, Kato \& Saegusa, 1982; Bornadiego, Díaz \& Marcos, 2019; Bornadiego, Díaz \& Marcos, 2015; Bornadiego, Díaz \& Marcos, 2014; Neo et al., 2013; Bornadiego, Díaz \& Marcos, 2019) and the high reactivity of 2-aminofuranes in Diels-Alder reactions (Padwa et al., 1997).

\section{MATERIALS \& METHODS}

\section{General synthetic techniques}

Materials. Methanol was dried by distillation over $\mathrm{CaH}_{2}$, immediately prior to use. Ethanol was freshly distilled from magnesium ethoxide, prepared from magnesium turnings in the presence of iodine. All other reagents were purchased from commercial sources and used as received.

Liquid reagents were measured using positive-displacement micropipettes with disposable tips and pistons. Thin layer chromatography was performed on aluminum plates, using $254 \mathrm{~nm}$ UV light or a mixture of $p$-anisaldehyde (2.5\%), acetic acid (1\%) and $\mathrm{H}_{2} \mathrm{SO}_{4}(3.4 \%)$ in $95 \%$ ethanol as developer. 


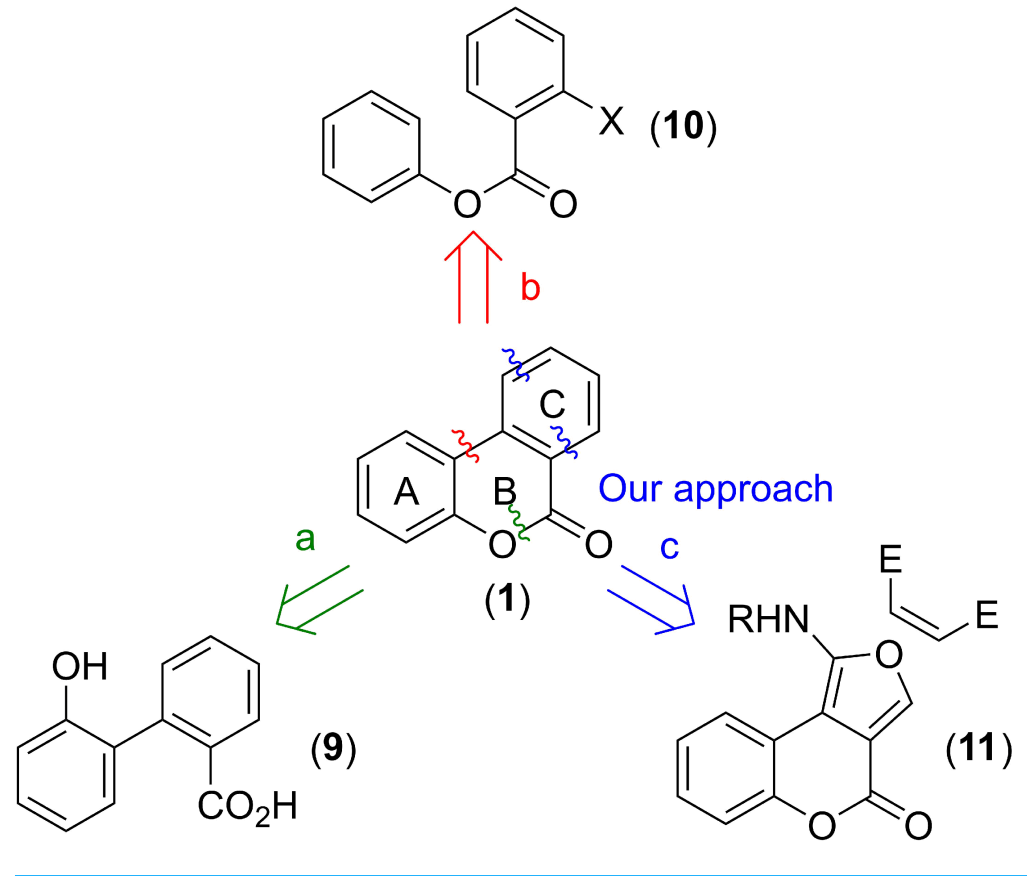

Figure 2 Strategies for the synthesis of benzo[c]coumarins.

Full-size DOI: $10.7717 /$ peerjochem.1/fig-2

Instrumentation. Melting points are uncorrected. IR spectra were recorded as $\mathrm{KBr}$ pellets. Proton and carbon-13 nuclear magnetic resonance $\left({ }^{1} \mathrm{H}\right.$ NMR or $\left.{ }^{13} \mathrm{C} \mathrm{NMR}\right)$ spectra were obtained on a $400 \mathrm{MHz}$ or $500 \mathrm{MHz}$ spectrometer. The assignments of signals in ${ }^{13} \mathrm{C}$ NMR were made by DEPT. High Resolution Mass Spectra (HRMS) were recorded using a 6520 Accurate Mass QTOF LC/MS Spectrometer. Experiments under microwave irradiation were performed in closed vials, using a focused single-mode microwave reactor CEM Discover BenchMate, provided with an IR internal thermal probe.

\section{Synthesis of 3-carbonylcoumarins (12a-d)}

Acyl acetate $16(3.3 \mathrm{mmol})$ and piperidine $(15 \% \mathrm{~mol})$ were added to a solution of salicylaldehyde (15, three mmol) in dry methanol (three $\mathrm{mL}$ ). The mixture was stirred at $\mathrm{rt}$ and the progress of the reaction was followed by tlc. After $24-72 \mathrm{~h}$ the solvent was evaporated and the resulting solid was washed with cold cyclohexane to give chromones (12a-d), which were used in the following reactions without further purification.

3-Benzoyl-2H-chromen-2-one (12a); (Specht, Martic \& Farid, 1982) (72 h, $661 \mathrm{mg}, 88 \%$ ). Obtained as a white solid; mp: $137-138^{\circ} \mathrm{C}$ (lit (Specht, Martic \& Farid, 1982) $137-139^{\circ} \mathrm{C}$ ); IR $\left(\mathrm{cm}^{-1}\right): 3408,1714,1657,1609,1567 ;{ }^{1} \mathrm{H}-\mathrm{NMR}\left(\mathrm{CDCl}_{3}, 500 \mathrm{MHz}\right) \delta 8.09(\mathrm{~s}, 1 \mathrm{H}), 7.90$ $(\mathrm{d}, J=7.23 \mathrm{~Hz}, 2 \mathrm{H}), 7.67-7.60(\mathrm{~m}, 3 \mathrm{H}), 7.49(\mathrm{t}, J=7.1 \mathrm{~Hz}, 2 \mathrm{H}), 7.42(\mathrm{~d}, J=8.1 \mathrm{~Hz}, 1 \mathrm{H})$, $7.36(\mathrm{t}, J=7.2 \mathrm{~Hz}, 1 \mathrm{H}) \mathrm{ppm} ;{ }^{13} \mathrm{C}\left\{{ }^{1} \mathrm{H}\right\}-\mathrm{NMR}\left(\mathrm{CDCl}_{3}, 101 \mathrm{MHz}\right) \delta 191.8(\mathrm{C}), 158.5(\mathrm{C})$, $154.9(\mathrm{C}), 145.6(\mathrm{CH}), 136.3(\mathrm{C}), 134.0(\mathrm{CH}), 133.8(\mathrm{CH}), 129.7(\mathrm{CH}), 129.3(\mathrm{CH}), 128.7$ $(\mathrm{CH}), 127.2(\mathrm{C}), 125.1(\mathrm{CH}), 118.3(\mathrm{C}), 117.1(\mathrm{CH}) \mathrm{ppm}$.

3-Benzoyl-6-bromo-2H-chromen-2-one (12b); (Wang et al., 2012) (24 h, $465 \mathrm{mg}, 47 \%$ ). Obtained as a white solid; mp: $171-176^{\circ} \mathrm{C}$ (lit (Wang et al., 2012) $171-172{ }^{\circ} \mathrm{C}$ ); IR $\left(\mathrm{cm}^{-1}\right)$ : 
3413, 3069, 1717, 1656, 1619, 1598; ${ }^{1} \mathrm{H}-\mathrm{NMR}\left(\mathrm{CDCl}_{3}, 500 \mathrm{MHz}\right) \delta 7.97(\mathrm{~s}, 1 \mathrm{H}), 7.87$ (d, $J=7.43 \mathrm{~Hz}, 2 \mathrm{H}), 7.72(\mathrm{~d}, J=7.13 \mathrm{~Hz}, 2 \mathrm{H}), 7.63(\mathrm{t}, J=7 \mathrm{~Hz}, 1 \mathrm{H}), 7.49$ (t, $J=7.4 \mathrm{~Hz}$, 2H), $7.30(\mathrm{~d}, J=9.06 \mathrm{~Hz}, 1 \mathrm{H}) \mathrm{ppm} ;{ }^{13} \mathrm{C}\left\{{ }^{1} \mathrm{H}\right\}-\mathrm{NMR}\left(\mathrm{CDCl}_{3}, 101 \mathrm{MHz}\right) \delta 191.2(\mathrm{C}), 157.8$ (C), $153.6(\mathrm{C}), 143.9(\mathrm{CH}), 136.4(\mathrm{CH}), 136.0(\mathrm{C}), 134.2(\mathrm{CH}), 131.4(\mathrm{CH}), 129.7(\mathrm{CH})$, $128.8(\mathrm{CH}), 128.2(\mathrm{C}), 119.8(\mathrm{C}), 118.8(\mathrm{CH}), 117.7(\mathrm{C}) \mathrm{ppm}$.

3-Acetyl-6-bromo-2H-chromen-2-one (12c); (Parveen, Khan \& Ahmed, 2019) (24 h, $360 \mathrm{mg}, 45 \%$ ). Obtained as a light yellow solid; mp: $224-228^{\circ} \mathrm{C}$ (lit (Parveen, Khan \& Ahmed, 2019). 232-233 $\left.{ }^{\circ} \mathrm{C}\right)$; IR ( $\left.\mathrm{cm}^{-1}\right): 3435,3042,1735,1675,1608,1550 ;{ }^{1} \mathrm{H}-\mathrm{NMR}$ $\left(\mathrm{CDCl}_{3}, 500 \mathrm{MHz}\right) \delta 8.40(\mathrm{~s}, 1 \mathrm{H}), 7.78(\mathrm{~s}, 1 \mathrm{H}), 7.73(\mathrm{~d}, J=8.27 \mathrm{~Hz}, 1 \mathrm{H}), 7.27(\mathrm{~s}, 1 \mathrm{H}), 2.72$ (s, 3H) ppm; ${ }^{13} \mathrm{C}\left\{{ }^{1} \mathrm{H}\right\}-\mathrm{NMR}\left(\mathrm{CDCl}_{3}, 126 \mathrm{MHz}\right) \delta 195.1$ (C), 158.7 (C), 154.3 (C), 146.1 $(\mathrm{CH}), 137.2(\mathrm{CH}), 132.3(\mathrm{CH}), 125.7(\mathrm{C}), 119.9(\mathrm{C}), 118.6(\mathrm{CH}), 117.7(\mathrm{C}), 30.6\left(\mathrm{CH}_{3}\right)$ ppm.

Ethyl 6-bromo-2-oxo-2H-chromene-3-carboxylate (12d); (Volmajer et al., 2005) (24 h, $197 \mathrm{mg}, 22 \%$ ). Obtained as a white solid; mp: $177^{\circ} \mathrm{C}$ (lit. $180-181^{\circ} \mathrm{C}$; (Volmajer et al., 2005) $172{ }^{\circ} \mathrm{C}$ (Santos-Contreras et al., 2007)); 3449, 3070, 2974, 1753, 1704, 1617, 1599; ${ }^{1} \mathrm{H}-\mathrm{NMR}\left(\mathrm{CDCl}_{3}, 500 \mathrm{MHz}\right) \delta 8.43(\mathrm{~s}, 1 \mathrm{H}), 7.75(\mathrm{~s}, 1 \mathrm{H}), 7.72(\mathrm{~d}, J=8.79 \mathrm{~Hz}, 1 \mathrm{H}), 7.25(\mathrm{~d}$, $J=8.79 \mathrm{~Hz}, 1 \mathrm{H}), 4.42(\mathrm{q}, J=7.12 \mathrm{~Hz}, 2 \mathrm{H}), 1.41(\mathrm{t}, J=7.11 \mathrm{~Hz}, 3 \mathrm{H}) \mathrm{ppm} ;{ }^{13} \mathrm{C}\left\{{ }^{1} \mathrm{H}\right\}-\mathrm{NMR}$ $\left(\mathrm{CDCl}_{3}, 126 \mathrm{MHz}\right) \delta 162.8(\mathrm{C}), 156.0(\mathrm{C}), 154.1(\mathrm{C}), 147.1(\mathrm{CH}), 137.0(\mathrm{CH}), 131.7(\mathrm{CH})$, $119.7(\mathrm{C}), 119.5(\mathrm{C}), 118.7(\mathrm{CH}), 117.5(\mathrm{C}), 62.3\left(\mathrm{CH}_{2}\right), 14.3\left(\mathrm{CH}_{3}\right) \mathrm{ppm}$.

\section{General procedure for the synthesis of $6 H$-benzo[c]chromen-6-ones (1a-n)}

Maleimide derivative $(14,0.36 \mathrm{mmol})$ and isocyanide $(13,0.36 \mathrm{mmol})$ were successively added to a solution of coumarin $(\mathbf{1 2}, 0.30 \mathrm{mmol}$ ) in dry ethanol (two $\mathrm{mL}$ ). The reaction mixture was stirred under $\mathrm{N}_{2}$ atmosphere, at $100{ }^{\circ} \mathrm{C}$ until completion, as judged by tlc. Then, $1 \mathrm{~N} \mathrm{HCl}(20 \mathrm{~mL})$ was added and the mixture stirred $1 \mathrm{~h}$ to hydrolyse any excess of isocyanide. The resulting mixture was extracted with $\mathrm{CH}_{2} \mathrm{Cl}_{2}$ and the organic phase was dried $\left(\mathrm{Na}_{2} \mathrm{SO}_{4}\right)$ and concentrated in the rotary evaporator. In the case of obtaining a solid, this was washed with cold cyclohexane to give the pure products $1 \mathbf{i}, 1 \mathbf{k}$ and 11 . In all the other cases, the crude was subjected to flash column chromatography $\left(\mathrm{SiO}_{2} 12 \mathrm{~g}\right.$ cartridge, cyclohexane to cyclohexane-ethyl acetate 8:2), to give the pure products $\mathbf{1 a - h} ; \mathbf{1 j}$ and $\mathbf{1 m}-\mathbf{n}$. 11-(Cyclohexylamino)-7,9-diphenylchromeno[3,4- $f]$ isoindole-6,8,10(9H)-trione (1a); (15 h, $124 \mathrm{mg}, 80 \%)$. Obtained as a fluorescent orange solid; mp: $243-244{ }^{\circ} \mathrm{C}$; IR $\left(\mathrm{cm}^{-1}\right)$ : $3311,2930,2850,1746,1706,1600,1501 ;{ }^{1} \mathrm{H}-\mathrm{NMR}\left(\mathrm{CDCl}_{3}, 500 \mathrm{MHz}\right) \delta 8.89$ (dd, $J=8.2$, $1.2 \mathrm{~Hz}, 1 \mathrm{H}), 7.51(\mathrm{t}, J=8.5 \mathrm{~Hz}, 1 \mathrm{H}), 7.47-7.42(\mathrm{~m}, 5 \mathrm{H}), 7.41-7.39(\mathrm{~m}, 2 \mathrm{H}), 7.38-7.34$ $(\mathrm{m}, 2 \mathrm{H}), 7.33(\mathrm{~m}, 1 \mathrm{H}), 7.30-7.26(\mathrm{~m}, 2 \mathrm{H}), 6.70(\mathrm{~d}, J=11.0 \mathrm{~Hz}, 1 \mathrm{H}), 3.43-3.27(\mathrm{~m}, 1 \mathrm{H})$, $1.90(\mathrm{~m}, 2 \mathrm{H}), 1.73(\mathrm{~m}, 2 \mathrm{H}), 1.39-1.30(\mathrm{~m}, 2 \mathrm{H}), 1.27-1.13(\mathrm{~m}, 4 \mathrm{H}) \mathrm{ppm} ;{ }^{13} \mathrm{C}\left\{{ }^{1} \mathrm{H}\right\}-\mathrm{NMR}$ $\left(\mathrm{CDCl}_{3}, 101 \mathrm{MHz}\right) \delta 168.7(\mathrm{C}), 164.7(\mathrm{C}), 158.6(\mathrm{C}), 150.4(\mathrm{C}), 145.6(\mathrm{C}), 136.3(\mathrm{C}), 136.2$ (C), $133.3(\mathrm{C}), 131.5(\mathrm{CH}), 131.2(\mathrm{C}), 129.1(\mathrm{CH}), 128.3(\mathrm{CH}), 128.2(\mathrm{CH}), 128.0(\mathrm{CH})$, $127.9(\mathrm{CH}), 126.6(\mathrm{CH}), 126.4(\mathrm{C}), 126.0(\mathrm{CH}), 124.1(\mathrm{CH}), 120.8(\mathrm{C}), 118.5(\mathrm{C}), 117.3$ $(\mathrm{CH}), 56.1(\mathrm{CH}), 33.9\left(\mathrm{CH}_{2}\right), 25.5\left(\mathrm{CH}_{2}\right), 24.8\left(\mathrm{CH}_{2}\right)$ ppm; HRMS (ESI ${ }^{+}$-QTOF) m/z: $[\mathrm{M}+\mathrm{H}]^{+}$Calcd for $\mathrm{C}_{33} \mathrm{H}_{27} \mathrm{~N}_{2} \mathrm{O}_{4}$ : 515.1966; found: 515.1975. 
2-Bromo-11-(cyclohexylamino)-7,9-diphenylchromeno[3,4- $f$ ]isoindole-6,8,10(9H)trione (1b); (23 h, $142 \mathrm{mg}, 79 \%$ ). Obtained as a fluorescent orange solid; mp: $142-146{ }^{\circ} \mathrm{C}$; IR $\left(\mathrm{cm}^{-1}\right): 3308,3061,2927,2851,1750,1707,1597 ;{ }^{1} \mathrm{H}-\mathrm{NMR}\left(\mathrm{CDCl}_{3}, 500 \mathrm{MHz}\right) \delta 9.11(\mathrm{~s}$, $1 \mathrm{H}), 7.60(\mathrm{dd}, J=8.7,2.3 \mathrm{~Hz}, 1 \mathrm{H}), 7.47-7.44(\mathrm{~m}, 2 \mathrm{H}), 7.44(\mathrm{~d}, J=2.5 \mathrm{~Hz}, 3 \mathrm{H}), 7.41-7.34$ $(\mathrm{m}, 3 \mathrm{H}), 7.26(\mathrm{dd}, J=5.3,4.2 \mathrm{~Hz}, 2 \mathrm{H}), 7.22(\mathrm{~d}, J=8.7 \mathrm{~Hz}, 1 \mathrm{H}), 6.69(\mathrm{~d}, J=11.1 \mathrm{~Hz}, 1 \mathrm{H})$, 3.36-3.25 (m, 1H), $1.97(\mathrm{~m}, 2 \mathrm{H}), 1.76(\mathrm{~m}, 2 \mathrm{H}), 1.42-1.33(\mathrm{~m}, 2 \mathrm{H}), 1.25(\mathrm{~m}, 4 \mathrm{H}) \mathrm{ppm}$; ${ }^{13} \mathrm{C}\left\{{ }^{1} \mathrm{H}\right\}-\mathrm{NMR}\left(\mathrm{CDCl}_{3}, 126 \mathrm{MHz}\right) \delta 168.5(\mathrm{C}), 164.6(\mathrm{C}), 158.0(\mathrm{C}), 149.4(\mathrm{C}), 145.6(\mathrm{C})$, $136.4(\mathrm{C}), 136.0(\mathrm{C}), 134.1(\mathrm{CH}), 131.8(\mathrm{C}), 131.2(\mathrm{C}), 129.1(\mathrm{CH}), 128.8(\mathrm{CH}), 128.5(\mathrm{C})$, $128.4(\mathrm{CH}), 128.2(\mathrm{CH}), 128.1(\mathrm{CH}), 126.6(\mathrm{CH}), 121.4(\mathrm{C}), 120.0(\mathrm{C}), 118.8(\mathrm{CH}), 117.1$ (C), $57.0(\mathrm{CH}), 33.9\left(\mathrm{CH}_{2}\right), 25.5\left(\mathrm{CH}_{2}\right), 24.9\left(\mathrm{CH}_{2}\right)$ ppm; HRMS (ESI ${ }^{+}$-QTOF) m/z: [M $+\mathrm{H}]^{+}$Calcd for $\mathrm{C}_{33} \mathrm{H}_{26} \mathrm{BrN}_{2} \mathrm{O}_{4}$ : 593.1071; found: 593.1093 .

11-(Cyclohexylamino)-9-methyl-7-phenylchromeno[3,4- $f$ ]isoindole-6,8,10(9H)-trione (1c); (59 h, $111 \mathrm{mg}, 82 \%)$. Obtained as a fluorescent yellow solid; mp: 199-205 ${ }^{\circ} \mathrm{C}$; IR $\left(\mathrm{cm}^{-1}\right): 3304,2924,2850,1745,1698,1611,1422 ;{ }^{1} \mathrm{H}-\mathrm{NMR}\left(\mathrm{CDCl}_{3}, 500 \mathrm{MHz}\right) \delta 8.90(\mathrm{~d}$, $J=8.2 \mathrm{~Hz}, 1 \mathrm{H}), 7.50(\mathrm{t}, J=7.6 \mathrm{~Hz}, 1 \mathrm{H}), 7.45(\mathrm{~m}, 3 \mathrm{H}), 7.32(\mathrm{~d}, J=8.0 \mathrm{~Hz}, 2 \mathrm{H}), 7.24(\mathrm{~m}$, $2 \mathrm{H}), 6.50(\mathrm{~d}, J=11.0 \mathrm{~Hz}, 1 \mathrm{H}), 3.29(\mathrm{~m}, 1 \mathrm{H}), 3.09(\mathrm{~s}, 3 \mathrm{H}), 1.87(\mathrm{~m}, 2 \mathrm{H}), 1.72(\mathrm{~m}, 2 \mathrm{H})$, $1.54(\mathrm{~m}, 1 \mathrm{H}), 1.37-1.11(\mathrm{~m}, 5 \mathrm{H}) \mathrm{ppm} ;{ }^{13} \mathrm{C}\left\{{ }^{1} \mathrm{H}\right\}-\mathrm{NMR}\left(\mathrm{CDCl}_{3}, 101 \mathrm{MHz}\right) \delta 169.6(\mathrm{C})$, $166.0(\mathrm{C}), 158.7$ (C), $150.5(\mathrm{C}), 145.1(\mathrm{C}), 136.5(\mathrm{C}), 135.8(\mathrm{C}), 133.2(\mathrm{C}), 131.4(\mathrm{CH})$, $128.2(\mathrm{CH}), 128.0(\mathrm{CH}), 127.9(\mathrm{CH}), 126.1(\mathrm{CH}), 125.9(\mathrm{C}), 124.1(\mathrm{CH}), 121.7(\mathrm{C}), 118.5$ (C), $117.2(\mathrm{CH}), 56.2(\mathrm{CH}), 33.9\left(\mathrm{CH}_{2}\right), 25.6\left(\mathrm{CH}_{2}\right), 24.9\left(\mathrm{CH}_{2}\right), 24.0\left(\mathrm{CH}_{3}\right)$ ppm; HRMS (ESI ${ }^{+}$-QTOF) m/z: $[\mathrm{M}+\mathrm{H}]^{+}$Calcd for $\mathrm{C}_{28} \mathrm{H}_{25} \mathrm{~N}_{2} \mathrm{O}_{4}$ : 453.1809; found: 453.1816 .

9-Methyl-11-(pentylamino)-7-phenylchromeno[3,4- $f$ ]isoindole-6,8,10(9H)-trione (1d); (59 h, $100 \mathrm{mg}, 76 \%)$. Obtained as a fluorescent orange solid; mp: $177-179{ }^{\circ} \mathrm{C}$; IR $\left(\mathrm{cm}^{-1}\right): 3346,2929,1737,1702,1609,1500 ;{ }^{1} \mathrm{H}-\mathrm{NMR}\left(\mathrm{CDCl}_{3}, 500 \mathrm{MHz}\right) \delta 8.69(\mathrm{~d}$, $J=8.23 \mathrm{~Hz}, 1 \mathrm{H}), 7.49(\mathrm{t}, J=8.2 \mathrm{~Hz}, 1 \mathrm{H}), 7.46-7.44(\mathrm{~m}, 3 \mathrm{H}), 7.33(\mathrm{t}, J=8.2 \mathrm{~Hz}, 2 \mathrm{H})$, 7.25-7.23 (m, 2H), 6.64 (bs, NH), $3.09(\mathrm{~m}, 5 \mathrm{H}), 1.62(\mathrm{q}, J=7.3 \mathrm{~Hz}, 2 \mathrm{H}), 1.32-1.24(\mathrm{~m}$, $4 \mathrm{H}), 0.89-0,86(\mathrm{~m}, 3 \mathrm{H}) \mathrm{ppm} ;{ }^{13} \mathrm{C}\left\{{ }^{1} \mathrm{H}\right\}-\mathrm{NMR}\left(\mathrm{CDCl}_{3}, 101 \mathrm{MHz}\right) \delta 169.6(\mathrm{C}), 166.1(\mathrm{C})$, $158.7(\mathrm{C}), 150.5(\mathrm{C}), 146.2(\mathrm{C}), 136.5(\mathrm{C}), 135.3(\mathrm{C}), 132.0(\mathrm{C}), 131.1(\mathrm{CH}), 128.5(\mathrm{C})$, $128.2(\mathrm{CH}), 128.0(\mathrm{CH}), 127.9(\mathrm{CH}), 126.4(\mathrm{CH}), 126.1(\mathrm{C}), 124.2(\mathrm{CH}), 120.2(\mathrm{C}), 118.3$ (C), 117.1 (CH), $49.4\left(\mathrm{CH}_{2}\right), 30.6\left(\mathrm{CH}_{2}\right), 29.0\left(\mathrm{CH}_{2}\right), 24.0\left(\mathrm{CH}_{3}\right), 22.5\left(\mathrm{CH}_{2}\right), 14.0\left(\mathrm{CH}_{3}\right)$ ppm; HRMS (ESI ${ }^{+}-\mathrm{QTOF}$ ) m/z: $[\mathrm{M}+\mathrm{H}]^{+}$Calcd for $\mathrm{C}_{28} \mathrm{H}_{24} \mathrm{~N}_{2} \mathrm{O}_{4}$ : 441.1809; found: 441.1830 .

11-((2,6-Dimethylphenyl)amino)-7-phenylchromeno[3,4- $f]$ isoindole-6,8,10(9H)trione (1e); (69 h, $43 \mathrm{mg}, 31 \%)$. Obtained as a fluorescent dark orange solid; mp: 310 $312{ }^{\circ} \mathrm{C}(\mathrm{dec})$; IR $\left(\mathrm{cm}^{-1}\right)$ : 3299, 1765, 1713, 1608, 1505; ${ }^{1} \mathrm{H}-\mathrm{NMR}\left(\mathrm{CDCl}_{3}, 500 \mathrm{MHz}\right) \delta$ $9.01(\mathrm{~s}, 1 \mathrm{H}), 7.86(\mathrm{~s}, 1 \mathrm{H}), 7.45(\mathrm{~m}, 3 \mathrm{H}), 7.29(\mathrm{~s}, 2 \mathrm{H}), 7.18(\mathrm{t}, J=7.5 \mathrm{~Hz}, 1 \mathrm{H}), 7.16-7.09$ $(\mathrm{m}, 2 \mathrm{H}), 6.89(\mathrm{~m}, 3 \mathrm{H}), 6.64(\mathrm{t}, J=7.5 \mathrm{~Hz}, 1 \mathrm{H}), 2.04(\mathrm{~s}, 6 \mathrm{H}) \mathrm{ppm} ;{ }^{13} \mathrm{C}\left\{{ }^{1} \mathrm{H}\right\}-\mathrm{NMR}\left(\mathrm{CDCl}_{3}\right.$, $101 \mathrm{MHz}) \delta 169.6(\mathrm{C}), 165.4(\mathrm{C}), 158.9(\mathrm{C}), 149.6(\mathrm{C}), 141.2(\mathrm{C}), 137.1(\mathrm{C}), 136.1(\mathrm{C})$, $135.2(\mathrm{CH}), 134.6(\mathrm{C}), 131.3(\mathrm{C}), 130.8(\mathrm{C}), 130.8(\mathrm{C}), 130.4(\mathrm{CH}), 129.3(\mathrm{CH}), 128.3(\mathrm{C})$, $128.7(\mathrm{CH}), 128.4(\mathrm{CH}), 128.0(\mathrm{CH}), 126.4(\mathrm{CH}), 126.3(\mathrm{CH}), 125.5(\mathrm{CH}), 122.1(\mathrm{CH})$, $117.5(\mathrm{C}), 116.1(\mathrm{CH}), 115.9(\mathrm{C}), 19.9\left(\mathrm{CH}_{3}\right) \mathrm{ppm}$; HRMS (ESI $\left.{ }^{-}-\mathrm{QTOF}\right) \mathrm{m} / \mathrm{z}:[\mathrm{M}-\mathrm{H}]^{-}$ Calcd for $\mathrm{C}_{29} \mathrm{H}_{19} \mathrm{~N}_{2} \mathrm{O}_{4}$ : 459.1350; found: 459.1365 . 
2-Bromo-11-(tert-butylamino)-7,9-diphenylchromeno[3,4- $f$ ]isoindole-6,8,10(9H)trione (1f); (>100 h, $19 \mathrm{mg}, 11 \%)$. Obtained as a fluorescent yellow solid; mp: 107-111 ${ }^{\circ} \mathrm{C}$; IR $\left(\mathrm{cm}^{-1}\right): 3446,2923,1769,1748,1710,1597 ;{ }^{1} \mathrm{H}-\mathrm{NMR}\left(\mathrm{CDCl}_{3}, 500 \mathrm{MHz}\right) \delta 10.11(\mathrm{~s}$, $1 \mathrm{H}), 7.58(\mathrm{~d}, J=8.7 \mathrm{~Hz}, 1 \mathrm{H}), 7.46-7.43(\mathrm{~m}, 5 \mathrm{H}), 7.38(\mathrm{~d}, J=7.5 \mathrm{~Hz}, 3 \mathrm{H}), 7.29(\mathrm{~m}, 2 \mathrm{H})$, $7.17(\mathrm{~d}, J=8.72 \mathrm{~Hz}, 1 \mathrm{H}), 5.83(\mathrm{~s}, 1 \mathrm{H}), 1.24(\mathrm{~s}, 9 \mathrm{H}) \mathrm{ppm} ;{ }^{13} \mathrm{C}\left\{{ }^{1} \mathrm{H}\right\}-\mathrm{NMR}\left(\mathrm{CDCl}_{3}, 101\right.$ $\mathrm{MHz}) \delta 167.4(\mathrm{C}), 164.4$ (C), 158.0 (C), 149.3 (C), 144.7 (C), 139.2 (C), 138.3 (C), 135.9 (C), $134.5(\mathrm{CH}), 131.1(\mathrm{C}), 130.2(\mathrm{CH}), 129.1(\mathrm{CH}), 128.5(\mathrm{CH}), 128.2(\mathrm{CH}), 128.1(\mathrm{CH})$, $128.0(\mathrm{CH}), 127.1(\mathrm{C}), 126.6(\mathrm{CH}), 125.7(\mathrm{C}), 121.6(\mathrm{C}), 118.9(\mathrm{CH}), 116.6(\mathrm{C}), 59.8$ (C), $30.1\left(\mathrm{CH}_{3}\right)$ ppm; HRMS (ESI $\left.{ }_{-} \mathrm{QTOF}\right) \mathrm{m} / \mathrm{z}$ : $[\mathrm{M}+\mathrm{H}]^{+}$Calcd for $\mathrm{C}_{31} \mathrm{H}_{24} \mathrm{BrN}_{2} \mathrm{O}_{4}$ : 567.0914; found: 567.0921 .

2-Bromo-9-methyl-11-(pentylamino)-7-phenylchromeno[3,4- $f$ ]isoindole-6,8,10(9H)trione (1g); (87 h, $98 \mathrm{mg}, 63 \%)$. Obtained as a fluorescent yellow solid; mp: $164-169^{\circ} \mathrm{C}$; IR $\left(\mathrm{cm}^{-1}\right): 3304,3072,2930,2858,1761,1744,1700,1609,1425 ;{ }^{1} \mathrm{H}-\mathrm{NMR}\left(\mathrm{CDCl}_{3}, 500\right.$ MHz) $\delta 8.86(\mathrm{~s}, 1 \mathrm{H}), 7.58(\mathrm{~d}, J=8.7 \mathrm{~Hz}, 1 \mathrm{H}), 7.47-7.44(\mathrm{~m}, 3 \mathrm{H}), 7.23-7.19(\mathrm{~m}, 3 \mathrm{H}), 6.65$ $(\mathrm{t}, J=6 \mathrm{~Hz}, 1 \mathrm{H}), 3.10(\mathrm{~s}, 3 \mathrm{H}), 3.08(\mathrm{t}, J=6.88 \mathrm{~Hz}, 2 \mathrm{H}), 1.68(\mathrm{q}, J=7.4 \mathrm{~Hz}, 2 \mathrm{H}), 1.37-1.31$ $(\mathrm{m}, 4 \mathrm{H}), 0.90(\mathrm{t}, J=7.1 \mathrm{~Hz}, 3 \mathrm{H}) \mathrm{ppm} ;{ }^{13} \mathrm{C}\left\{{ }^{1} \mathrm{H}\right\}-\mathrm{NMR}\left(\mathrm{CDCl}_{3}, 126 \mathrm{MHz}\right) \delta 169.4(\mathrm{C})$, $165.9(\mathrm{C}), 158.1(\mathrm{C}), 149.4(\mathrm{C}), 146.2(\mathrm{C}), 136.2(\mathrm{C}), 135.6(\mathrm{C}), 133.8(\mathrm{CH}), 130.6(\mathrm{C})$, $129.5(\mathrm{C}), 129.0(\mathrm{CH}), 120.2(\mathrm{CH}), 128.0(\mathrm{CH}), 128.0(\mathrm{CH}), 126.2(\mathrm{C}), 120.8(\mathrm{C}), 119.9$ (C), $118.8(\mathrm{CH}), 117.2(\mathrm{C}), 49.7\left(\mathrm{CH}_{2}\right), 30.5\left(\mathrm{CH}_{2}\right), 29.1\left(\mathrm{CH}_{2}\right), 24.1\left(\mathrm{CH}_{3}\right), 22.5\left(\mathrm{CH}_{2}\right)$, $14.1\left(\mathrm{CH}_{3}\right)$ ppm; HRMS (ESI ${ }^{+}$-QTOF) $[\mathrm{M}+\mathrm{H}]^{+}$Calcd for $\mathrm{C}_{27} \mathrm{H}_{24} \mathrm{BrN}_{2} \mathrm{O}_{4}$ : 519.0914; found: 519.0918 .

2-Bromo-11-((2,6-dimethylphenyl)amino)-7-phenylchromeno[3,4- $f$ ]isoindole-

6,8,10(9H)-trione (1h); $(67 \mathrm{~h}, 50 \mathrm{mg}, 31 \%)$. Obtained as an orange solid; mp: $322-325^{\circ} \mathrm{C}$ (dec); IR $\left(\mathrm{cm}^{-1}\right): 3319,1765,1737,1710,1607,1477 ;{ }^{1} \mathrm{H}-\mathrm{NMR}\left(\mathrm{CDCl}_{3}, 500 \mathrm{MHz}\right) \delta 8.96$ (s, 1H), $7.77(\mathrm{~s}, 1 \mathrm{H}), 7.46(\mathrm{~m}, 3 \mathrm{H}), 7.28-7.25(\mathrm{~m}, 4 \mathrm{H}), 7.00-6.92(\mathrm{~m}, 4 \mathrm{H}), 2.07(\mathrm{~s}, 6 \mathrm{H})$ ppm; ${ }^{13} \mathrm{C}\left\{{ }^{1} \mathrm{H}\right\}$-NMR $\left(\mathrm{CDCl}_{3}, 101 \mathrm{MHz}\right) \delta 169.4(\mathrm{C}), 165.3$ (C), $158.3(\mathrm{C}), 148.5$ (C), 141.4 (C), $136.7(\mathrm{C}), 135.8(\mathrm{C}), 134.6(\mathrm{C}), 133.2(\mathrm{CH}), 131.1(\mathrm{C}), 130.0(\mathrm{CH}), 129.5(\mathrm{C}), 129.3$ $(\mathrm{CH}), 129.2(\mathrm{C}), 128.4(\mathrm{CH}), 128.2(\mathrm{CH}), 128.1(\mathrm{CH}), 126.4(\mathrm{C}), 125.9(\mathrm{CH}), 117.8(\mathrm{C})$, $117.6(\mathrm{C}), 117.4(\mathrm{C}), 115.8(\mathrm{C}), 20.1\left(\mathrm{CH}_{3}\right)$ ppm; HRMS (ESI ${ }^{-}$-QTOF) [M - H] ${ }^{-}$Calcd for $\mathrm{C}_{29} \mathrm{H}_{18} \mathrm{BrN}_{2} \mathrm{O}_{4}$ : 537.0455; found: 537.0428.

2-Bromo-11-(cyclohexylamino)-7-methyl-9-phenylchromeno[3,4- $f$ ]isoindole-

6,8,10(9H)-trione (1i); (7 h, $158 \mathrm{mg}, 99 \%)$. Obtained as an orange solid; mp: $241-244{ }^{\circ} \mathrm{C}$; IR $\left(\mathrm{cm}^{-1}\right): 3439,2930,2853,1758,1698,1598,1500 ;{ }^{1} \mathrm{H}-\mathrm{NMR}\left(\mathrm{CDCl}_{3}, 500 \mathrm{MHz}\right) \delta 9.16$ $(\mathrm{s}, 1 \mathrm{H}), 7.59(\mathrm{~d}, J=8.65 \mathrm{~Hz}, 1 \mathrm{H}), 7.54(\mathrm{t}, J=7.6 \mathrm{~Hz}, 2 \mathrm{H}), 7.46-7.42(\mathrm{~m}, 3 \mathrm{H}), 7.23$ (d, $J=8.7 \mathrm{~Hz}, 1 \mathrm{H}), 6.50(\mathrm{~d}, J=11.14 \mathrm{~Hz}, 1 \mathrm{H}), 3.14(\mathrm{~m}, 4 \mathrm{H}), 1.89(\mathrm{~m}, 2 \mathrm{H}), 1.72-1.18(\mathrm{~m}$, $8 \mathrm{H}) \mathrm{ppm} ;{ }^{13} \mathrm{C}\left\{{ }^{1} \mathrm{H}\right\}-\mathrm{NMR}\left(\mathrm{CDCl}_{3}, 101 \mathrm{MHz}\right) \delta 168.4(\mathrm{C}), 166.5(\mathrm{C}), 159.2(\mathrm{C}), 149.2(\mathrm{C})$, $144.3(\mathrm{C}), 136.4(\mathrm{C}), 134.0(\mathrm{CH}), 132.4(\mathrm{C}), 131.3(\mathrm{C}), 129.3(\mathrm{CH}), 128.9(\mathrm{CH}), 128.6$ $(\mathrm{CH}), 128.3(\mathrm{C}), 127.3(\mathrm{C}), 126.7(\mathrm{CH}), 121.9(\mathrm{C}), 120.1(\mathrm{C}), 118.6(\mathrm{CH}), 117.1(\mathrm{C}), 56.8$ $(\mathrm{CH}), 33.8\left(\mathrm{CH}_{2}\right), 25.5\left(\mathrm{CH}_{2}\right), 24.9\left(\mathrm{CH}_{2}\right), 16.2\left(\mathrm{CH}_{3}\right)$ ppm; HRMS (ESI ${ }^{+}$-QTOF) [M + $\mathrm{H}]^{+}$Calcd for $\mathrm{C}_{28} \mathrm{H}_{24} \mathrm{BrN}_{2} \mathrm{O}_{4}$ : 531.0914; found: 531.0885 .

2-Bromo-11-(tert-butylamino)-7,9-dimethylchromeno[3,4- $f$ ]isoindole-6,8,10(9H)trione (1j); (27 h, $8 \mathrm{mg}, 6 \%)$. Obtained as a fluorescent yellow solid; mp: $210-213^{\circ} \mathrm{C}$; IR $\left(\mathrm{cm}^{-1}\right): 3305,2960,1760,1741,1697,1433 ;{ }^{1} \mathrm{H}-\mathrm{NMR}\left(\mathrm{CDCl}_{3}, 500 \mathrm{MHz}\right) \delta 10.11(\mathrm{~s}, 1 \mathrm{H})$, 
$7.55(\mathrm{~d}, J=8.72 \mathrm{~Hz}, 1 \mathrm{H}), 7.16(\mathrm{~d}, J=8.71 \mathrm{~Hz}, 1 \mathrm{H}), 5.52(\mathrm{~s}, 1 \mathrm{H}), 3.19(\mathrm{~s}, 3 \mathrm{H}), 3.14(\mathrm{~s}$, $3 \mathrm{H}), 1.13$ (s, 9H) ppm; ${ }^{13} \mathrm{C}\left\{{ }^{1} \mathrm{H}\right\}-\mathrm{NMR}\left(\mathrm{CDCl}_{3}, 126 \mathrm{MHz}\right) \delta 168.9$ (C), 167.5 (C), 159.3 (C), $149.2(\mathrm{C}), 142.6(\mathrm{C}), 138.8(\mathrm{C}), 138.4(\mathrm{C}), 134.2(\mathrm{CH}), 130.6(\mathrm{CH}), 128.6(\mathrm{C}), 128.4$ (C), $126.2(\mathrm{C}), 121.7(\mathrm{C}), 118.6(\mathrm{CH}), 116.5(\mathrm{C}), 59.1(\mathrm{C}), 29.9\left(\mathrm{CH}_{3}\right), 24.2\left(\mathrm{CH}_{3}\right), 16.4$ $\left(\mathrm{CH}_{3}\right)$ ppm; HRMS (ESI ${ }^{+}$-QTOF) $[\mathrm{M}+\mathrm{H}]^{+}$Calcd for $\mathrm{C}_{21} \mathrm{H}_{20} \mathrm{BrN}_{2} \mathrm{O}_{4}$ : 443.0601; found: 443.0585 .

2-Bromo-7,9-dimethyl-11-(pentylamino)chromeno[3,4- $f$ ]isoindole-6,8,10(9H)-trione (1k); (13 h, $72 \mathrm{mg}, 52 \%)$. Obtained as a fluorescent orange solid; mp: $139-142{ }^{\circ} \mathrm{C}$; IR $\left(\mathrm{cm}^{-1}\right): 3324,2931,1740,1698,1606,1436 ;{ }^{1} \mathrm{H}-\mathrm{NMR}\left(\mathrm{CDCl}_{3}, 500 \mathrm{MHz}\right) \delta 8.91(\mathrm{~s}, 1 \mathrm{H})$, $7.56(\mathrm{~d}, J=8.58 \mathrm{~Hz}, 1 \mathrm{H}), 7.21(\mathrm{~d}, J=8.67 \mathrm{~Hz}, 1 \mathrm{H}), 6.45(\mathrm{t}, J=5.3 \mathrm{~Hz}, 1 \mathrm{H}), 3.19(\mathrm{~s}, 3 \mathrm{H})$, $3.08(\mathrm{~s}, 3 \mathrm{H}), 3.01-2.96(\mathrm{~m}, 2 \mathrm{H}), 1.61(\mathrm{~m}, 2 \mathrm{H}), 1.31-1.30(\mathrm{~m}, 4 \mathrm{H}), 0.88(\mathrm{~m}, 3 \mathrm{H}) \mathrm{ppm}$; ${ }^{13} \mathrm{C}\left\{{ }^{1} \mathrm{H}\right\}$-NMR $\left(\mathrm{CDCl}_{3}, 101 \mathrm{MHz}\right) \delta 169.3$ (C), 167.7 (C), 159.3 (C), 149.2 (C), 145.0 (C), $135.4(\mathrm{C}), 133.7(\mathrm{CH}), 131.2(\mathrm{C}), 129.0(\mathrm{CH}), 128.9(\mathrm{C}), 126.9(\mathrm{C}), 121.4(\mathrm{C}), 119.9$ (C), $118.5(\mathrm{CH}), 117.2(\mathrm{C}), 49.5\left(\mathrm{CH}_{2}\right), 30.3\left(\mathrm{CH}_{2}\right), 29.0\left(\mathrm{CH}_{2}\right), 24.1\left(\mathrm{CH}_{3}\right), 22.5\left(\mathrm{CH}_{2}\right)$, $16.2\left(\mathrm{CH}_{3}\right), 14.0\left(\mathrm{CH}_{3}\right)$ ppm; HRMS (ESI $\left.{ }_{-} \mathrm{QTOF}\right)[\mathrm{M}+\mathrm{H}]^{+}$Calcd for $\mathrm{C}_{22} \mathrm{H}_{22} \mathrm{BrN}_{2} \mathrm{O}_{4}$ : 457.0758; found: 457.0755 .

11-(Benzylamino)-2-bromo-7-methylchromeno[3,4- $f$ ]isoindole-6,8,10(9H)-trione (11); (18 h, $107 \mathrm{mg}, 77 \%)$. Obtained as a fluorescent orange solid; mp: $234-238{ }^{\circ} \mathrm{C}$; IR $\left(\mathrm{cm}^{-1}\right): 3431,1757,1737,1709,1606,1424 ;{ }^{1} \mathrm{H}-\mathrm{NMR}\left(\mathrm{CDCl}_{3}, 500 \mathrm{MHz}\right) \delta 9.02(\mathrm{~s}$, $1 \mathrm{H}), 7.78(\mathrm{~s}, 1 \mathrm{H}), 7.54(\mathrm{~d}, J=8.66 \mathrm{~Hz}, 1 \mathrm{H}), 7.24-7.17(\mathrm{~m}, 4 \mathrm{H}), 7.12(\mathrm{~d}, J=6.96 \mathrm{~Hz}$, 2H), $6.73(\mathrm{t}, J=6.3 \mathrm{~Hz}, 1 \mathrm{H}), 4.14(\mathrm{~d}, J=6.49 \mathrm{~Hz}, 2 \mathrm{H}), 3.02(\mathrm{~s}, 3 \mathrm{H}) \mathrm{ppm} ;{ }^{13} \mathrm{C}\left\{{ }^{1} \mathrm{H}\right\}-\mathrm{NMR}$ $\left(\mathrm{CDCl}_{3}, 101 \mathrm{MHz}\right) \delta 168.5(\mathrm{C}), 167.0(\mathrm{C}), 159.1$ (C), $149.4(\mathrm{C}), 144.6(\mathrm{C}), 137.5(\mathrm{C})$, $136.8(\mathrm{C}), 134.2(\mathrm{CH}), 131.9(\mathrm{C}), 129.2(\mathrm{C}), 129.1(\mathrm{CH}), 129.1(\mathrm{CH}), 128.1(\mathrm{CH}), 127.9$ $(\mathrm{CH}), 127.4(\mathrm{C}), 122.4(\mathrm{C}), 119.8(\mathrm{C}), 118.7(\mathrm{CH}), 117.5(\mathrm{C}), 52.9\left(\mathrm{CH}_{2}\right), 16.2\left(\mathrm{CH}_{3}\right) \mathrm{ppm}$; HRMS (ESI ${ }^{+}$-QTOF) Calcd for $\mathrm{C}_{23} \mathrm{H}_{15} \mathrm{BrN}_{2} \mathrm{O}_{4} \mathrm{H}^{+}$: 463.0288 ; found: 463.0289 .

2-Bromo-11-((2,6-dimethylphenyl)amino)-7-methylchromeno[3,4- $f$ ]isoindole-

6,8,10(9H)-trione (1m); (18 h, $60 \mathrm{mg}, 42 \%)$. Obtained as a fluorescent orange solid; mp: 337-342 (dec); IR (cm $\left.{ }^{-1}\right)$ : 3441, 2919, 2850, 1739, 1713, 1634; ${ }^{1} \mathrm{H}-\mathrm{NMR}\left(\mathrm{CDCl}_{3}\right.$, $500 \mathrm{MHz}) \delta 8.77(\mathrm{~s}, 1 \mathrm{H}), 7.72(\mathrm{~s}, 1 \mathrm{H}), 7.30(\mathrm{~s}, 1 \mathrm{H}), 7.01(\mathrm{~d}, J=8.69 \mathrm{~Hz}, 1 \mathrm{H}), 6.95-6.88$ $(\mathrm{m}, 4 \mathrm{H}), 3.12(\mathrm{~s}, 3 \mathrm{H}), 2.01(\mathrm{~s}, 6 \mathrm{H}) \mathrm{ppm}$; due to the insolubility of compound $\mathbf{1 m}$, it was not possible to record its ${ }^{13} \mathrm{C}-\mathrm{NMR}$ spectrum. HRMS (ESI ${ }^{-}$-QTOF) $[\mathrm{M}-\mathrm{H}]^{-}$Calcd for $\mathrm{C}_{24} \mathrm{H}_{16} \mathrm{BrN}_{2} \mathrm{O}_{4}$ : 475.0299; found: 475.0326 .

2-Bromo-11-((4-methoxyphenyl)amino)-7-methylchromeno[3,4- $f$ ]isoindole6,8,10(9H)-trione (1n); (32 h, $78 \mathrm{mg}, 54 \%)$. Obtained as a light red oil; IR $\left(\mathrm{cm}^{-1}\right)$ : 3441, 2919, 2850, 1759, 1732, 1702, 1509; ${ }^{1} \mathrm{H}-\mathrm{NMR}\left(\mathrm{CDCl}_{3}, 500 \mathrm{MHz}\right) \delta 8.54(\mathrm{~s}, 1 \mathrm{H})$, $8.42(\mathrm{~d}, J=2.26 \mathrm{~Hz}, 1 \mathrm{H}), 7.73(\mathrm{~s}, 1 \mathrm{H}), 7.37(\mathrm{~d}, J=8.7 \mathrm{~Hz}, 1 \mathrm{H}), 7.09(\mathrm{~d}, J=8.69 \mathrm{~Hz}, 1 \mathrm{H})$, 6.75-6.68 (m, $4 \mathrm{H}), 3.71(\mathrm{~s}, 3 \mathrm{H}), 3.16(\mathrm{~s}, 3 \mathrm{H}) \mathrm{ppm}$; due to the insolubility of compound 1n, it was not possible to record its ${ }^{13} \mathrm{C}$-NMR spectrum. HRMS $\left(\mathrm{ESI}^{+}\right.$-QTOF) $[\mathrm{M}+\mathrm{H}]^{+}$ Calcd for $\mathrm{C}_{23} \mathrm{H}_{16} \mathrm{BrN}_{2} \mathrm{O}_{5}$ : 479.0237; found: 479.0234.

\section{RESULTS AND DISCUSSION}

Aminofurans are very reactive dienes that are known to undergo Diels-Alder cycloadditions in relatively mild reaction conditions. We have recently shown that 2-aminofurans, 
<smiles>[R]N=C1OC([R])=C2C(=O)Oc3ccc([R]#CCC#[R])cc3C12</smiles>

12

17

11
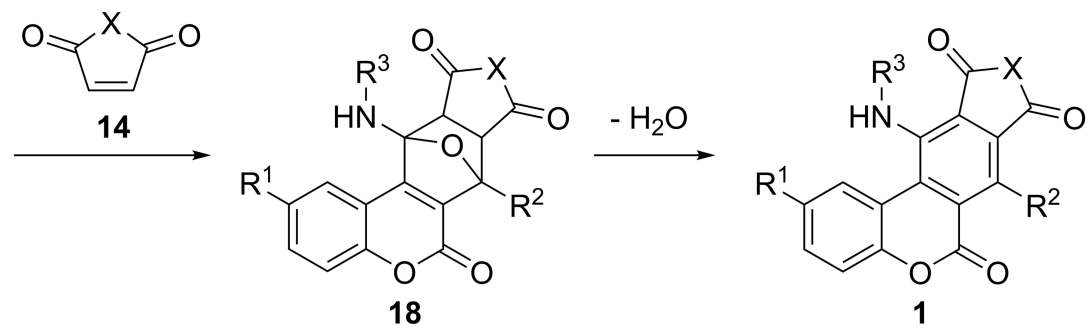

Figure 3 Schematic representation of sequential $[4+1]$ and $[4+2]$ cycloaddition reactions leading to the synthesis of 3-carbonylcoumarins.

Full-size DOI: 10.7717/peerjochem.1/fig-3

readily synthesized by a [4+1] cycloaddition of isocyanides and $\alpha, \beta$-unsaturated carbonyl compounds, can react with dienophiles in a single operation to give anilines (Bornadiego, Díaz \& Marcos, 2019; Bornadiego, Díaz \& Marcos, 2015; Bornadiego, Díaz \& Marcos, 2014; Neo et al., 2013; Bornadiego, Díaz \& Marcos, 2019). Henceforth, we reason that 1-amino$4 H$-furo[3,4-c]chromen-4-ones (11) would be suitable intermediate dienes for the DielsAlder construction benzo $[c]$ coumarin $C$ ring. Accordingly, we propose a multicomponent synthesis of benzo[c]coumarins (1) by the sequential [4+1]/[4+2] cycloaddition of isocyanides (13), 3-carbonylcoumarins (12) and dienophiles (14, Fig. 3).

The starting carbonylcoumarins (12) were synthesized by the Knoevenagel condensation of salicylaldehydes (15) with different $\beta$-ketoesters (16), followed by cyclization, according to slightly modified Farid's method (Specht, Martic \& Farid, 1982). In our case, the reaction was optimally carried out at room temperature in methanol, using piperidine as basic catalyst (Fig. 4).

In order to prove our strategy for the synthesis of benzo[c]coumarins $(\mathbf{1})$, we reacted 3-benzoylcoumarin 12a with 1.2 equivalents of cyclohexyl isocyanide 13a and $\mathrm{N}$-phenylmaleimide 14a in THF. After $12 \mathrm{~h}$ at $25^{\circ} \mathrm{C}$, the reaction medium became yelloworange and a new highly fluorescent yellow product was evident on tlc. However, the reaction was extraordinarily sluggish at room temperature and it did not reach completion even after 30 days (Table 1, entry 1). The reaction was still slow when the temperature was raised to $80^{\circ} \mathrm{C}$, but it did conclude after 9 days, allowing the isolation of a new product, 1a, the identity of which was confirmed by ${ }^{1} \mathrm{H}-\mathrm{RMN},{ }^{13} \mathrm{C}-\mathrm{RMN}$, IR and MS (Table 1 , entry 2 ).

With the purpose of finding better reaction conditions, we explored the use of different solvents, temperatures, and the addition of catalysts (Table 1). Using a solvent with a higher boiling point, such as dibutyl ether, resulted in a complex mixture, difficult to purify. Fortunately, when we carried out the reaction in ethanol at $100^{\circ} \mathrm{C}$, benzocoumarin 1a was obtained in just $15 \mathrm{~h}$ with an $80 \%$ yield (Table 1, entry 5 ). Similarly, the reaction 


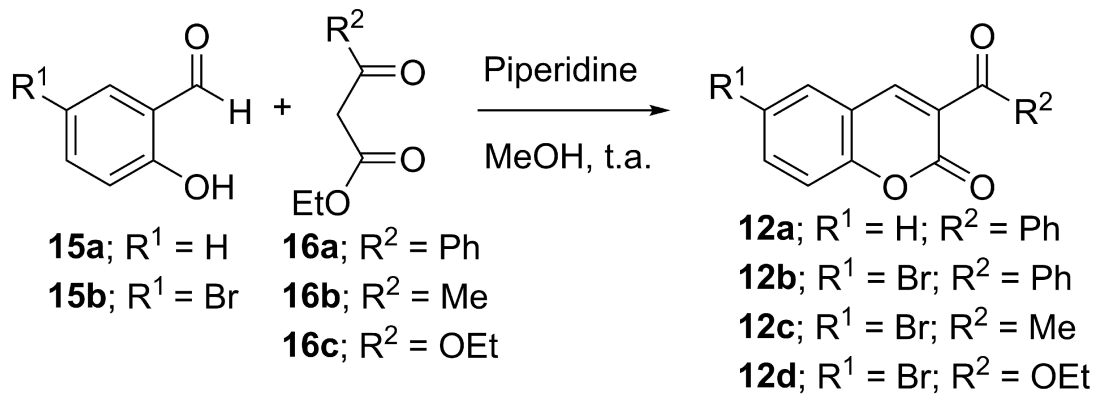

Figure 4 Synthesis of 3-carbonylcoumarins.

Table 1 Optimization of the 3-component reaction.

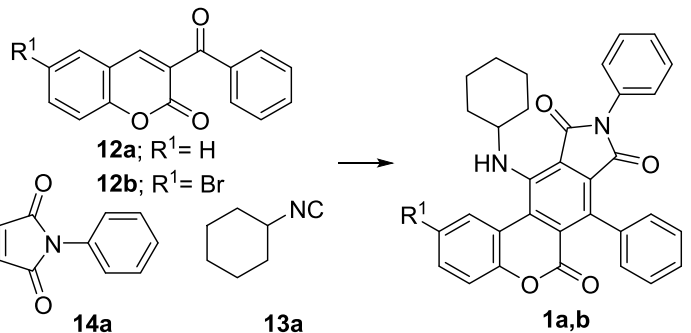

\begin{tabular}{clllll} 
Entry & Solvent & $\mathbf{T},{ }^{\circ} \mathbf{C}$ & $\begin{array}{l}\text { Catalyst, } \\
\text { \% mol }\end{array}$ & Time & $\begin{array}{l}\text { Yield, } \\
\text { \% (Product) }\end{array}$ \\
\hline 1 & $\mathrm{THF}$ & $\mathrm{rt}$ & - & $30 \mathrm{~d}$ & $\mathrm{IR}^{\mathrm{a}}$ \\
2 & $\mathrm{THF}$ & 80 & - & $9 \mathrm{~d}$ & $44(\mathbf{1 a})$ \\
3 & $\mathrm{THF}$ & 80 & $\mathrm{Y}(\mathrm{OTf})_{3}, 5$ & $9 \mathrm{~d}$ & $\mathrm{CM}^{\mathrm{b}}$ \\
4 & $\mathrm{Bu}{ }_{2} \mathrm{O}$ & 150 & - & $43 \mathrm{~h}$ & $\mathrm{CM}^{\mathrm{b}}$ \\
5 & $\mathrm{EtOH}$ & 100 & - & $15 \mathrm{~h}$ & $80(\mathbf{1 a})$ \\
6 & $\mathrm{EtOH}$ & $\mathrm{rt}$ & & $30 \mathrm{~d}$ & $32(\mathbf{1 b})$ \\
7 & $\mathrm{EtOH}$ & 100 & & $23 \mathrm{~h}$ & $79(\mathbf{1 b})$ \\
8 & $\mathrm{EtOH}$ & 100 & $\mathrm{H}_{2} \mathrm{SO}_{4}, 20$ & $65 \mathrm{~h}$ & $74(\mathbf{1 b})$ \\
9 & $\mathrm{EtOH}$ & 100 & $\mathrm{TU}^{\mathrm{c}}, 20$ & $27 \mathrm{~h}$ & $75(\mathbf{1 b})$ \\
\hline
\end{tabular}

Notes.

${ }^{\mathrm{a}} \mathrm{IR}$, incomplete reaction.

${ }^{\mathrm{b}} \mathrm{CM}$, complex mixture.

${ }^{\mathrm{c}} \mathrm{TU}, 1,3$-bis(3,5-bis(trifluoromethyl)phenyl)thiourea.

with bromocoumarin $\mathbf{1 2 b}$ afforded the corresponding benzocoumarin $\mathbf{1 b}$ with a $79 \%$ yield (entry 7). In consequence, the reaction, which is very slow in THF, seems to be favored in ethanol, suggesting the occurrence of charged intermediates that may be stabilized in protic polar solvents. This is in accordance with our theoretical studies ( $W u, X u \& X i e$, 2005) that show that related syntheses of 2 -aminofuranes proceed through a [4+1] stepwise cycloaddition involving a zwitterionic intermediate (Bornadiego, Díaz \& Marcos, 2019). On 


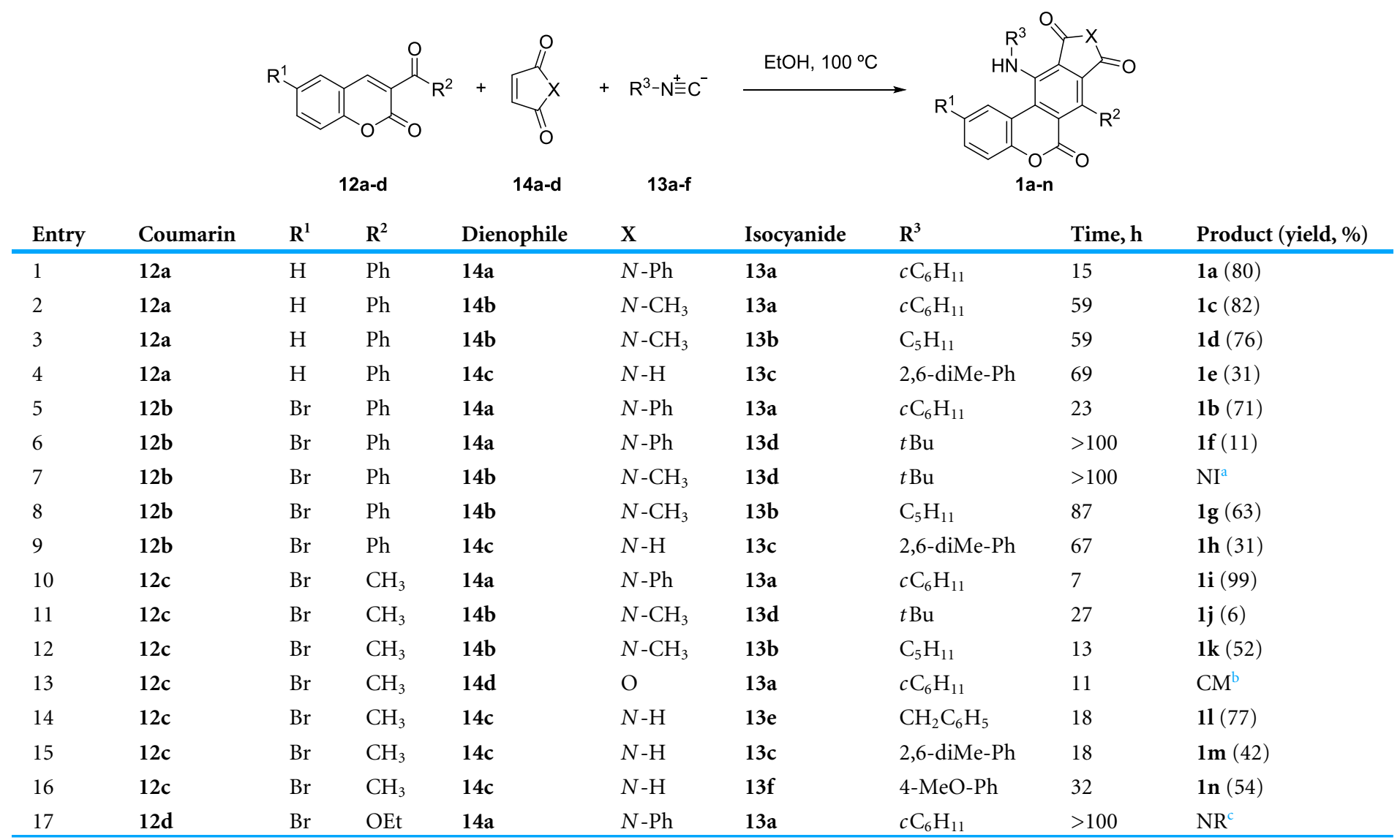

Notes.

${ }^{\mathrm{a}} \mathrm{NI}$, non isolated.

${ }^{\mathrm{b}} \mathrm{CM}$, complex mixture.

${ }^{\mathrm{c}} \mathrm{NR}$, no reaction.

the other hand, the use of proton donors (entries 8 and 9) or Lewis acid catalysts (entry 3 ) did not significantly improve the reaction.

Thus, the optimal reaction conditions were applied to different combinations of isocyanides (13a-f), dienophiles (14a-d) and 3-carbonylcoumarins (12a-d; Table 2).

In most of the cases the products are obtained with moderate to excellent yields. The reaction proceeds equally well with different aromatic and aliphatic substituted 3-carbonylcoumarins. Conversely, as expected, coumarin ester 12d (Table 2, entry 17) does not react. $N$-substituted and non-substituted maleimides can be used as dienophiles; however, the reaction with maleic anhydride produces a complex mixture of products (entry 13). Aliphatic isocyanides (entries $1-3,5,8,10,12,14$ ) require shorter reaction times and result in better yields than less reactive aromatic isocyanides (entries 4, 9, 15, 16). An exception is tert-butyl isocyanide (entries 6, 7, 11), possibly due to steric hindrance. 


\section{CONCLUSIONS}

In conclusion, we have successfully designed a highly convergent and atom-economic synthesis of benzo $[c]$ coumarins. Our strategy is based on the trapping with dienophiles of reactive 2-aminofurans generated in a [4+1] cycloaddition of isocyanides and readily available 3-carbonyl coumarins. In this way, the target compounds are readily obtained in one-pot, in mild conditions with no need of catalysis. Furthermore, different substitution patterns can be easily accessed, as the reaction tolerates a wide choice of the three starting materials. Therefore, this multicomponent approach provides a flexible method to fine tune the properties of the products.

\section{ADDITIONAL INFORMATION AND DECLARATIONS}

\section{Funding}

This work was supported by the Junta de Extremadura and European Regional Development Fund (IB16095). The funders had no role in study design, data collection and analysis, decision to publish, or preparation of the manuscript.

\section{Grant Disclosures}

The following grant information was disclosed by the authors:

Junta de Extremadura and European Regional Development Fund: IB16095.

\section{Competing Interests}

Carlos Fernández Marcos is an Academic Editor for PeerJ.

\section{Author Contributions}

- Ana Bornadiego performed the experiments, analyzed the data, prepared figures and/or tables, approved the final draft.

- Jesús Díaz conceived and designed the experiments, analyzed the data, contributed reagents/materials/analysis tools, prepared figures and/or tables, authored or reviewed drafts of the paper, approved the final draft.

- Carlos Fernández Marcos conceived and designed the experiments, analyzed the data, contributed reagents/materials/analysis tools, prepared figures and/or tables, authored or reviewed drafts of the paper, approved the final draft.

\section{Data Availability}

The following information was supplied regarding data availability:

Raw data are available in the Supplemental Files.

\section{Supplemental Information}

Supplemental information for this article can be found online at http://dx.doi.org/10.7717/ peerj-ochem.1\#supplemental-information. 


\section{REFERENCES}

Borges F, Roleira F, Milhazes N, Santana L, Uriarte E. 2005. Simple coumarins and analogues in medicinal chemistry: occurrence, synthesis and biological activity. Current Medicinal Chemistry 12(8):887-916 DOI 10.2174/0929867053507315.

Bornadiego A, Díaz J, Marcos CF. 2019. Tandem synthesis of 4-aminoxanthones is controlled by a water-assisted tautomerization: a general straightforward reaction. Organic and Biomolecular Chemistry 17(6):1410-1422 DOI 10.1039/C8OB02527D.

Bornadiego A, Díaz J, Marcos CF. 2014. Synthesis of 4-aminoxanthones by an uncatalyzed, multicomponent reaction. Advanced Synthesis \& Catalysis 356(4):718-722 DOI 10.1002/adsc.201300750.

Bornadiego A, Díaz J, Marcos CF. 2015. Regioselective tandem [4+1]-[4+2] synthesis of amino-substituted dihydroxanthones and xanthones. Journal of Organic Chemistry 80(12):6165-6172 DOI 10.1021/acs.joc.5b00658.

Bornadiego A, Díaz J, Marcos CF. 2019. Tandem synthesis of polycyclic isoindoles. Journal of Organic Chemistry 84(11):7426-7433 DOI 10.1021/acs.joc.9b00381.

Ceci C, Lacal PM, Tentori L, De Martino MG, Miano R, Graziani G. 2018. Experimental evidence of the antitumor, antimetastatic and antiangiogenic activity of ellagic acid. Nutrients 10(11):1756-1779 DOI 10.3390/nu10111756.

Chatani N, Oshita M, Tobisu M, Ishii Y, Murai S. 2003. A $\mathrm{GaCl}_{3}$-Catalyzed [4+1] Cycloaddition of alpha,beta-unsaturated carbonyl compounds and isocyanides leading to unsaturated gamma-lactone derivatives. Journal of the American Chemical Society 125(26):7812-7813 DOI 10.1021/ja035014u.

François G, Timperman G, Holenz J, Assi LA, Geuder T, Maes L, Dubois J, Hanocq M, Bringmannj G. 2016. Naphthylisoquinoline alkaloids exhibit strong growthinhibiting activities against Plasmodium falciparum and P. berghei in vitroStructure-activity relationships of dioncophylline C. Annals of Tropical Medicine \& Parasitology 90(2):115-123 DOI 10.1080/00034983.1996.11813035.

Garazd YL, Garazd MM. 2016. Natural dibenzo[ $b, d]$ pyran-6-ones: structural diversity and biological activity. Chemistry of Natural Compounds 52(1):1-18 DOI 10.1007/s10600-016-1536-4.

He Y, Zhang X, Cui L, Wang J, Fan X. 2012. Catalyst-free synthesis of diversely substituted 6C-benzo $[c]$ chromenes and $6 H$-benzo $[c]$ chromen-6-ones in aqueous media under MWI. Green Chemistry 14(12):3429-3435 DOI 10.1039/c2gc36379h.

Ito $\mathrm{Y}$, Kato H, Saegusa T. 1982. A new approach for stereoselective synthesis of gammabutyrolactones. Journal of Organic Chemistry 47(4):741-743

DOI 10.1021/jo00343a030.

Khanolkar AD, Lu D, Ibrahim M, Duclos Jr RI, Thakur GA, Malan Jr TP, Porreca F, Veerappan V, Tian X, George C, Parrish DA, Papahatjis DP, Makriyannis A. 2007. Cannabilactones: a novel class of CB2 selective agonists with peripheral analgesic activity. Journal of Medicinal Chemistry 50(26):6493-6500 DOI 10.1021/jm070441u.

Lavoie S, Sweeney-Jones AM, Mojib N, Dale B, Gagaring K, McNamara CW, Quave CL, Soapi K, Kubanek J. 2019. Antibacterial oligomeric polyphenols from the 
green alga Cladophora socialis. Journal of Organic Chemistry 84(9):5035-5045 DOI 10.1021/acs.joc.8b03218.

Luo S, Luo FX, Zhang XS, Shi ZJ. 2013. Synthesis of dibenzopyranones through palladium-catalyzed directed C-H activation/carbonylation of 2-arylphenols. Angewandte Chemie International Edition in English 52(40):10598-10601 DOI 10.1002/anie.201304295.

Mao Z, Sun W, Fu L, Luo H, Lai D, Zhou L. 2014. Natural dibenzo-alpha-pyrones and their bioactivities. Molecules 19(4):5088-5108 DOI 10.3390/molecules19045088.

Matson JA, Rose WC, Bush JA, Myllymaki R, Bradner WT, Doyle TW. 1989. Antitumor activity of chrysomycins M and V. The Journal of Antibiotics 42(9):1446-1448 DOI 10.7164/antibiotics.42.1446.

Medina FG, Marrero JG, Macias-Alonso M, Gonzalez MC, Cordova-Guerrero I, Teissier Garcia AG, Osegueda-Robles S. 2015. Coumarin heterocyclic derivatives: chemical synthesis and biological activity. Natural Products Reports 32(10):1472-1507 DOI 10.1039/c4np00162a.

Morack T, Metternich JB, Gilmour R. 2018. Vitamin catalysis: direct, photocatalytic synthesis of benzocoumarins via (-)-riboflavin-mediated electron transfer. Organic Letters 20(5):1316-1319 DOI 10.1021/acs.orglett.8b00052.

Neo AG, Bornadiego A, Díaz J, Marcaccini S, Marcos CF. 2013. Elusive 2-aminofuran Diels-Alder substrates for a straightforward synthesis of polysubstituted anilines. Organic and Biomolecular Chemistry 11(38):6546-6555 DOI 10.1039/c3ob41411f.

Ortiz Villamizar MC, Zubkov FI, Puerto Galvis CE, Vargas Méndez LY, Kouznetsov VV. 2017. The study of metal-free and palladium-catalysed synthesis of benzochromenes via direct $\mathrm{C}-\mathrm{H}$ arylation using unactivated aryl benzyl ethers derived from essential oils as raw materials. Organic Chemistry Frontiers 4(9):1736-1744 DOI 10.1039/C7QO00232G.

Padwa A, Dimitroff M, Waterson AG, Wu TH. 1997. Diels-Alder reaction of 2-aminosubstituted furans as a method for preparing substituted anilines. Journal of Organic Chemistry 62(12):4088-4096 DOI 10.1021/j09702599.

Parveen I, Khan D, Ahmed N. 2019. Regioselective hydrodehalogenation of aromatic $\alpha$ and $\beta$-halo carbonyl compounds by $\mathrm{CuI}$ in isopropanol. European Journal of Organic Chemistry 2019(4):759-764 DOI 10.1002/ejoc.201801385.

Pottie IR, Nandaluru PR, Benoit WL, Miller DO, Dawe LN, Bodwell GJ. 2011. Synthesis of $6 \mathrm{H}$-dibenzo[ $b, d]$ pyran-6-ones using the inverse electron demand Diels-Alder reaction. Journal of Organic Chemistry 76(21):9015-9030 DOI 10.1021/jo201775e.

Ramirez NP, Bosque I, Gonzalez-Gomez JC. 2015. Photocatalytic dehydrogenative lactonization of 2-arylbenzoic acids. Organic Letters 17(18):4550-4553 DOI 10.1021/acs.orglett.5b02269.

Santos-Contreras RJ, Martinez-Martinez FJ, Garcia-Baez EV, Padilla II M, Peraza AL, Hopfl H. 2007. Carbonyl-carbonyl, carbonyl-pi and carbonyl-halogen dipolar 
interactions as the directing motifs of the supramolecular structure of ethyl 6-chloro2-oxo- $2 H$-chromene-3-carboxylate and ethyl 6-bromo-2-oxo- $2 H$-chromene-3carboxylate. Acta Crystallographica. Section C: Crystal Structure Communications 63(pt 4):o239-o242 DOI 10.1107/S1600536806052767.

Solfrizzo M. 2017. Recent advances on Alternaria mycotoxins. Current Opinion in Food Science 17:57-61 DOI 10.1016/j.cofs.2017.09.012.

Specht DP, Martic PA, Farid S. 1982. Ketocoumarins: a new class of triplet sensitizers. Tetrahedron 38(9):1203-1211 DOI 10.1016/0040-4020(82)85104-1.

Stefanachi A, Leonetti F, Pisani L, Catto M, Carotti A. 2018. Coumarin: a natural, privileged and versatile scaffold for bioactive compounds. Molecules 23(2):250-283 DOI 10.3390/molecules23020250.

Tomita F, Takahashi K-I, Tamaoki T. 1982. Gilvocarcins, new antitumor antibiotics. 4. Mode of action. The Journal of Antibiotics 35(8):1038-1041

DOI 10.7164/antibiotics.35.1038.

Trenor SR, Shultz AR, Love BJ, Long TE. 2004. Coumarins in polymers: from light harvesting to photo-cross-linkable tissue scaffolds. Chemical Reviews 104(6):3059-3077 DOI 10.1021/cr030037c.

Vogel A. 1820. Darstellung von Benzoesäure aus der Tonka-Bohne und aus den Meliloten - oder Steinklee - Blumen. Annalen der Physik 64(2):161-166 DOI 10.1002/andp.18200640205.

Volmajer J, Toplak R, Leban I, Marechal AML. 2005. Synthesis of new iminocoumarins and their transformations into N-chloro and hydrazono compounds. Tetrahedron 61(29):7012-7021 DOI 10.1016/j.tet.2005.05.020.

Wagner BD. 2009. The use of coumarins as environmentally-sensitive fluorescent probes of heterogeneous inclusion systems. Molecules 14(1):210-237 DOI 10.3390/molecules14010210.

Wang Y, Yu Z-H, Zheng H-F, Shi D-Q. 2012. DABCO and Bu3P catalyzed [4+2] and [3 + 2] cycloadditions of 3-acyl-2H-chromen-ones and ethyl 2, 3-butadienoate. Organic and Biomolecular Chemistry 10(38):7739-7746 DOI 10.1039/c2ob26300a.

Wu Y, Xu K, Xie D. 2005. A computational study on the mechanism for the $\mathrm{GaCl}_{3}$ catalyzed [4+1] cycloaddition of $\alpha \beta$-unsaturated ketone and 2,6-dimethylphenyl isocyanide. Tetrahedron 61(2):507-512 DOI 10.1016/j.tet.2004.10.068.

Yamashita N, Shin-Ya K, Furihata K, Hayakawa Y, Seto H. 1998. New ravidomycin analogues, FE35A and FE35B, apoptosis inducers produced by Streptomyces rochei. The Journal of Antibiotics 51(12):1105-1108 DOI 10.7164/antibiotics.51.1105. 